\title{
Storing free magnetic energy in the solar corona
}

\author{
G. Vekstein $\dagger$ \\ Jodrell Bank Centre for Astrophysics, School of Physics and Astronomy, University of Manchester, \\ Manchester M13 9PL, UK
}

(Received 22 April 2016; revised 27 July 2016; accepted 28 July 2016)

This article presents a mini-tutorial aimed at a wide readership not familiar with the field of solar plasma physics. The exposition is centred around the issue of excess/free magnetic energy stored in the solar corona. A general consideration is followed with a particular example of coronal magnetic arcade, where free magnetic energy builds up by photospheric convective flows. In the context of solar physics the major task is to explain how this free energy can be released quickly enough to match what is observed in coronal explosive events such as solar flares. Therefore, in the last section of the paper we discuss briefly a possible role of magnetic reconnection in these processes. This is done in quite simple qualitative physical terms, so that an interested reader can follow it up in more detail with help of the provided references.

\section{Introduction}

The Sun, our nearest star, is a very interesting physical object. One of its mysteries, not yet completely cracked, is the solar corona: very hot $\left(T_{c} \sim 10^{6} \mathrm{~K}\right)$ plasma several thousand kilometres above the visible solar surface (the photosphere). The puzzle is why the corona, while being more distant than the photosphere from the ultimate energy source (which is nuclear fusion reactions in the Sun's interior), has a temperature much higher than the photosphere $\left(T_{p h} \approx 5800 \mathrm{~K}\right)$. The corona is visible during total solar eclipses as a faint glow around the solar disk when it is covered by the Moon. Although records of eclipses go back millennia, the corona as a separate object has been reported only since the 17 th century. Then, during the eclipse of 1869, an unknown green emission line was detected in the coronal spectrum. Since, just a year earlier, another unknown optical line emitted from the Sun had been attributed to a new chemical element named helium, it was tempting to follow suit by introducing another new element, 'coronium'.

Although helium was identified in a terrestrial laboratory in 1895 , in the case of 'coronium' the story turned out to be more complicated. It was not until the 1930s that it was realized that the line under discussion is actually produced by highly ionized iron. This immediately implied that the corona is made of a hot plasma with the temperature exceeding $10^{6} \mathrm{~K}$. Therefore, what is visible around the solar disk during eclipses is mere photospheric light scattered by coronal electrons. The emission of the corona itself falls into the ultraviolet (UV) and soft X-ray bands, and, therefore, can be observed only by space-borne instruments. Thus, state-of-the-art

$\dagger$ Email address for correspondence: g.vekstein@manchester.ac.uk 

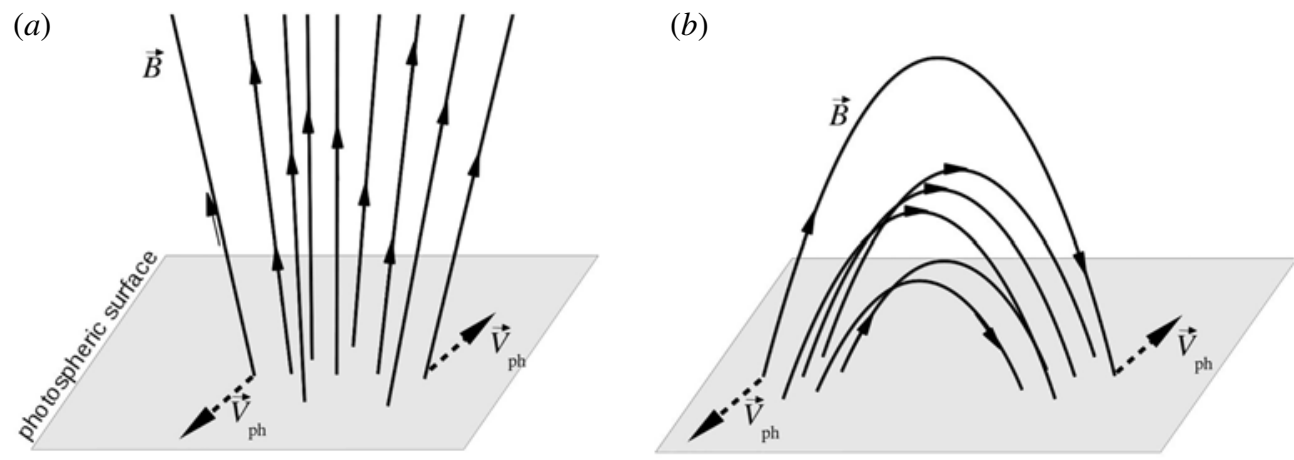

FIgURE 1. Basic elements of the coronal magnetic field. (a) A coronal hole;

(b) a coronal loop.

images of the corona, made by the Solar Dynamics Observatory launched in 2010, are available in Main Gallery-SDO/Solar Dynamics Observatory-NASA: sdo.gsfc.nasa.gov/gallery/main. Their distinctive feature is the presence of numerous fine structures, which are a signature of the underlying coronal magnetic field. This field is strong enough to supress the motion of electrons and protons (hydrogen is the main element of the coronal plasma) across magnetic field lines. Indeed, under a typical coronal field strength $B_{c} \sim 100 \mathrm{G}$ and plasma density $n_{c} \sim 10^{9} \mathrm{~cm}^{-3}$ (see, e.g. Golub \& Pasachoff 2010), the gyrofrequency of protons is $\omega_{B}^{(p)}=e B / m_{p} c \sim 10^{6} \mathrm{~s}^{-1}$, while the collision frequency of thermal protons with energy $E_{p} \sim k T_{c} \sim 10^{2} \mathrm{eV}$ is approximately $v_{p} \sim 1 \mathrm{~s}^{-1}$. Thus, when $v_{p} \ll \omega_{B}^{(p)}$, protons (as well as much lighter plasma electrons) are strongly magnetized, and their gyroradius, which is of the order of $\rho_{B}^{(p)} \sim\left(\left(\sqrt{k T / m_{p}}\right) / \omega_{B}^{(p)}\right) \sim 10 \mathrm{~cm}$, is negligibly small in the coronal context.

However, guiding charged particles along magnetic field lines is by far not the only role played by the coronal magnetic field. Under the above mentioned coronal parameters, the magnetic energy per unit volume, $W_{B}=B_{c}^{2} / 8 \pi$, is much larger than the plasma thermal energy $W_{T} \sim n_{c} k T_{c}$. Their ratio is commonly known as a nondimensional parameter plasma $\beta, \beta \equiv 8 \pi n k T / B^{2}$. In coronal active regions, which look mostly bright in X-ray images of the Sun, $\beta \sim 10^{-2}$. Therefore, the interaction of the solar magnetic field with plasma should be essential for the very existence of the solar corona. Nowadays, it is well known that the Sun is not unique in this respect as X-ray emission associated with coronae is detected from a large number of solar-type stars. However, understanding the mechanism of coronae formation is not only of academic interest. The solar corona is a source of the solar wind, which is the continuous plasma outflow from the Sun that extends far beyond the orbits of the Earth and other planets (see, e.g. Meyer-Vernet 2007). Therefore, what is going on in the corona (solar flares, coronal mass ejections, etc.) determines what is presently called 'Space Weather' and the resulting terrestrial effects (such as, for example, magnetospheric substorms). Many interesting details about the history of solar coronal studies, as well as the present-day hot topics in this research field, can be found in an excellent book by Golub \& Pasachoff (2010).

\section{Coronal magnetic field and free magnetic energy}

Two basic 'building blocks' of the coronal magnetic field are schematically drawn in figure 1. The first one, in diagram $(a)$, is the so-called coronal hole. In this case 
only one end (the footpoint) of a magnetic field line is anchored to the photospheric surface, and the field line extends into the heliosphere. In UV and X-ray images of the Sun, coronal holes look like dim areas on the solar disk because of a low plasma density (charged particles easily escape upward along the magnetic field lines). On the other hand, a bipolar coronal loop, as shown in diagram $(b)$, has both magnetic footpoints connected to the photosphere. Thus, it is a natural plasma trap, and bright regions in the corona are made of such loops. The photospheric surface in figure 1 may be considered a sharp boundary between tenuous low- $\beta$ coronal plasma and dense sub-photospheric fluid. The energetics of the latter is dominated by the kinetic energy of the convective flow with a velocity of $V_{p h} \sim 1 \mathrm{~km} \mathrm{~s}^{-1}$ and a granule size of $l_{p h} \sim 10^{3} \mathrm{~km}$. Given this, the photospheric granular turnover time is $\tau_{p h} \sim l_{p h} / V_{p h} \sim$ $10^{3} \mathrm{~s}$. This granular flow continuously shuffles footpoints of the coronal magnetic field as shown in figure 1. In the case of coronal holes, these generate magnetohydrodynamic (mainly Alfvén) waves that propagate upward along magnetic field lines, ultimately providing energy input to the solar wind. A very different response comes from the coronal loops. In a low- $\beta$ plasma such as the coronal one, where the dynamics is governed by the magnetic field, perturbations in a system propagate with the Alfvén velocity $V_{A}=B / \sqrt{4 \pi n m_{p}}$. With the coronal parameters, one gets $V_{A}^{(c)} \sim 10^{3} \mathrm{~km} \mathrm{~s}^{-1}$, which, for a coronal loop of a typical length $L_{c} \sim 10^{9} \mathrm{~cm}$, yields the dynamical time scale of $\tau_{A}^{(c)}=\left(L_{c} / V_{A}^{(c)}\right) \sim 10 \mathrm{~s}$. Thus, $\tau_{p h} \gg \tau_{A}^{(c)}$, which means that such photospheric perturbations are quasistatic. Therefore, the coronal loop remains close to a state of magnetostatic equilibrium at any one time. In a low- $\beta$ plasma, where thermal pressure forces are small, this equilibrium requires vanishing magnetic force, $\boldsymbol{F}_{B}=(\boldsymbol{j} \times \boldsymbol{B}) / c \approx 0$, which is the case if electric current $\boldsymbol{j}=c(\boldsymbol{\nabla} \times \boldsymbol{B}) / 4 \pi$ flows along magnetic field lines, i.e.

$$
(\nabla \times \boldsymbol{B})=\alpha(\boldsymbol{r}) \boldsymbol{B} .
$$

This equation defines the so-called 'force-free magnetic field'. Note that since $\nabla \cdot \boldsymbol{j}=$ $\boldsymbol{\nabla} \cdot \boldsymbol{B}=0$, the function $\alpha(\boldsymbol{r})$ in (2.1) should be constant along a magnetic field line but, in a general case may vary from one field line to another. These electric currents are the source of an excess (free) magnetic energy, which is responsible for coronal heating and all other manifestations of coronal activity.

To put the issue of the free magnetic energy on a firm quantitative footing, consider a planar magnetic field shown in figure 2(a). This is clearly a non-potential field, which is evident from the presence of closed magnetic field lines (hence, $\oint B \cdot \mathrm{d} l \neq$ $0 \Rightarrow \nabla \times \boldsymbol{B} \neq 0 \Rightarrow \boldsymbol{j} \neq 0$ ). In fact, this is a superposition of the potential $\mathrm{X}$-point magnetic configuration (see figure $2 b$ ) generated by some remote electric currents and the azimuthal field due to an axial electric current concentrated around the X-point (which transforms it into the magnetic O-point).

Assume now that some process of magnetic relaxation takes place inside the area encircled in figure 2(a). For example, it could be just simple Ohmic dissipation of the central electric current. As far as the exterior of the relaxation circle is concerned, suppose that it is filled with a perfectly conducting and very heavy fluid. Therefore, during the internal magnetic relaxation, this fluid remains immobile because of its strong inertia, so the external magnetic field does not change. What is the minimum of the magnetic energy $W_{M}$ inside the circle that can be achieved under such relaxation? Since

$$
W_{M}=\int_{V} \frac{B^{2}}{8 \pi} \mathrm{d} V,
$$


(a)

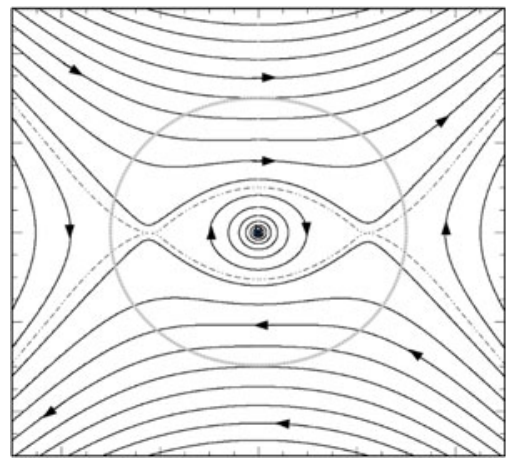

(b)

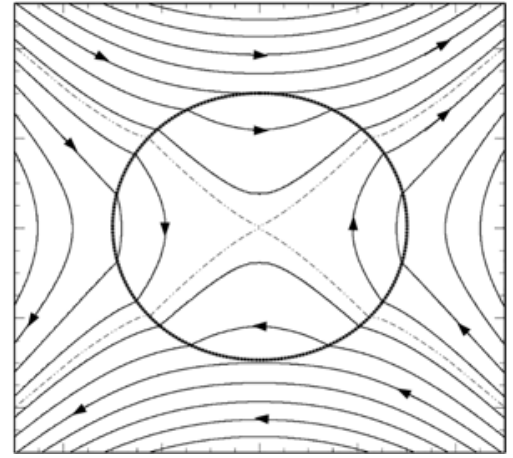

FIGURE 2. Internal magnetic relaxation. (a) Initial magnetic configuration; (b) relaxed state with a potential magnetic field inside the encircled area. Fine dash-dotted lines indicate 'separatrices', which are boundaries between domains of different magnetic field lines connectivity.

the integrand in (2.2) is always non-negative, and at first glance one may conclude that the sought after minimum corresponds simply to $\boldsymbol{B} \equiv 0$ everywhere inside the relaxation volume. However, such a state cannot be reached by the internal magnetic relaxation while the external magnetic field remains unchanged. Indeed, since $\boldsymbol{\nabla} \cdot \boldsymbol{B}=0$, the normal component of $\boldsymbol{B}$ must be continuous at the boundary surface. Therefore, only magnetic fields with a prescribed boundary condition $\left.B_{n}\right|_{S}=\left.B_{n}^{(e x t)}\right|_{S}$ are allowed to compete in minimizing the magnetic energy (2.2). (Note that since $\nabla \cdot \boldsymbol{B}=0$, the condition $\int_{S} B_{n}^{(e x t)} \mathrm{d} S=0$ is always satisfied.) The right solution can be guessed with the help of the following qualitative physical consideration. Suppose that inside the relaxation domain there is a finite electric resistivity $\eta$, which results in the Ohmic dissipation power per unit volume being equal to $\eta j^{2} \geqslant 0$. Since the dissipated energy is tapped from the magnetic energy, the latter will decrease with time until $\boldsymbol{j}=0$ everywhere in the domain, i.e. when the magnetic field there becomes potential. By representing this potential field as $\boldsymbol{B}_{p}=\nabla \phi$, and adding the necessary condition $\nabla \cdot \boldsymbol{B}=0$, the magnetic potential $\phi$ should be a solution of the Laplace equation $\nabla^{2} \phi=0$ with the boundary condition $\partial \phi /\left.\partial n\right|_{S}=B_{n}^{(e x t)}$. This is the so-called Neumann problem, a classical problem in mathematical physics, and it always has a unique solution (see, e.g. Kannenberg 1989 and references therein). Given this, any magnetic field admissible in the domain under consideration can be represented as $\boldsymbol{B}=\boldsymbol{B}_{p}+\boldsymbol{b}$ with the boundary condition $\left.b_{n}\right|_{S}=0$. Inserting this expression for $\boldsymbol{B}$ into (2.2) yields

$$
W_{M}=\frac{1}{8 \pi} \int_{V}\left(B_{p}^{2}+b^{2}+2 \boldsymbol{B}_{p} \cdot \boldsymbol{b}\right) \mathrm{d} V .
$$

Since $\boldsymbol{\nabla} \cdot \boldsymbol{b}=0$, the last term in the above expression for $W_{M}$ can be shown to vanish, as

$$
\int_{V}\left(\boldsymbol{B}_{p} \cdot \boldsymbol{b}\right) \mathrm{d} V=\int_{V}(\nabla \phi \cdot \boldsymbol{b}) \mathrm{d} V=\int_{V} \nabla(\phi \boldsymbol{b}) \mathrm{d} V=\int_{S} \phi b_{n} \mathrm{~d} S=0 .
$$

Thus, the potential magnetic field corresponds to the state of minimum magnetic energy. Any deviation from this potential field, $\boldsymbol{b}$, which is associated with a non-zero electric current, results in excess magnetic energy $\Delta W_{M}$ given by

$$
\Delta W_{M}=\frac{1}{8 \pi} \int_{V} b^{2} \mathrm{~d} V .
$$


Returning to the example of figure 2 , the respective relaxed state with a potential magnetic field is depicted in figure $2(b)$. Note that such internal magnetic relaxation is not just a simple elimination of the central electric current. A surface current is induced instead at the boundary surface, which is evident from the discontinuity there of the tangential magnetic field component. Furthermore, there is an apparent change between the initial and the final magnetic configurations. Under the relaxation process, magnetic field lines are allowed to break up and reconnect, resulting in the simplification of the magnetic topology. Such a process, known as 'magnetic reconnection', plays an essential role in laboratory and space plasmas. In the context of the solar corona it is briefly discussed in $\S 4$.

\section{Energetics of the coronal magnetic arcade}

As explained above, force-free magnetic fields, which are defined by (2.1), are at the heart of the solar coronal magnetohydrodynamics. In a real three-dimensional (3-D) solar corona their structure could be quite complicated (Amari et al. 1997). Therefore, here we explore a simplified model, where the magnetic field possesses all three spatial components but is invariant with respect to one coordinate (say, y). Any such magnetic field can be represented as

$$
\boldsymbol{B}(x, z)=\left[\nabla \Psi(x, z) \times \boldsymbol{e}_{y}\right]+B_{y}(x, z) \boldsymbol{e}_{y},
$$

where $\boldsymbol{e}_{y}$ is a unit vector along the $y$-axis. This form ensures that $\boldsymbol{\nabla} \cdot \boldsymbol{B}=0$. The function $\Psi(x, z)$ here is called 'the poloidal flux function', while $B_{y}$ is a toroidal field component. This terminology originates from the fusion-oriented research with toroidal laboratory devices. Equation $\Psi(x, z)=$ const. determines projections of magnetic field lines on the $(x, z)$-plane. Such a projection is defined by the equation $\mathrm{d} x / B_{x}=\mathrm{d} z / B_{z}$, and since $B_{x}=-\partial \Psi / \partial z, B_{z}=\partial \Psi / \partial x,(\partial \Psi / \partial x) \mathrm{d} x+(\partial \Psi / \partial z) \mathrm{d} z=\mathrm{d} \Psi=0$ along this line. The geometrical meaning of $\Psi$ becomes evident from calculating the amount of poloidal magnetic flux, $\Delta \Psi_{p}$, contained in a 2-D flux tube bounded by two poloidal field lines defined respectively by $\Psi(x, z)=\Psi_{1}$ and $\Psi(x, z)=\Psi_{2}$ as shown in figure 3 .

$$
\begin{aligned}
\Delta \Psi_{p} & =\int_{1}^{2} \mathrm{~d} l B_{p} \sin \theta=\boldsymbol{e}_{y} \cdot \int_{1}^{2}\left(\mathrm{~d} \boldsymbol{l} \times \boldsymbol{B}_{p}\right)=\boldsymbol{e}_{y} \cdot \int_{1}^{2} \mathrm{~d} \boldsymbol{l} \times\left(\nabla \Psi \times \boldsymbol{e}_{y}\right) \\
& =-\int_{1}^{2} \mathrm{~d} \boldsymbol{l} \cdot \nabla \Psi=\Psi_{1}-\Psi_{2} .
\end{aligned}
$$

Consider now what condition is required to make the field in (3.1) force free. Thus, according to (3.1),

$$
(\nabla \times \boldsymbol{B})=\left[\nabla B_{y}(x, z) \times \boldsymbol{e}_{y}\right]-\nabla^{2} \Psi(x, z) \boldsymbol{e}_{y} .
$$

Since for a force-free field vectors (3.1) and (3.3) are parallel, $\nabla \Psi$ should be parallel to $\nabla \boldsymbol{B}_{y}$, i.e. $B_{y}(x, z) \equiv B_{y}(\Psi)$. This implies that function $\alpha(\boldsymbol{r})$ introduced in (2.1) is equal to $\alpha(\boldsymbol{r})=\alpha(\Psi)=\mathrm{d} B_{y}(\Psi) / \mathrm{d} \Psi$. Furthermore, since the ratio of the other components of vectors (3.1) and (3.3) should be equal to the ratio of their poloidal components, $-\nabla^{2} \Psi=\alpha B_{y}$, i.e. $\nabla^{2} \Psi+B_{y}(\Psi)\left(\mathrm{d} B_{y} / \mathrm{d} \Psi\right)=0$. This equation is known in magnetohydrodynamics as the 'Grad-Shafranov equation' for a force-free magnetic field. In general, this is a nonlinear equation that reduces to a much simpler linear 


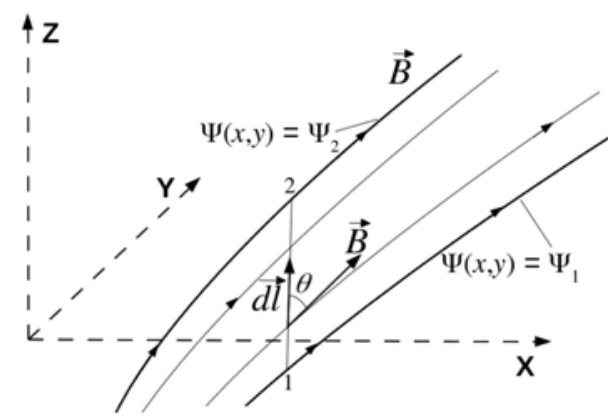

FIGURE 3. On the geometrical meaning of the poloidal flux function.

(a)

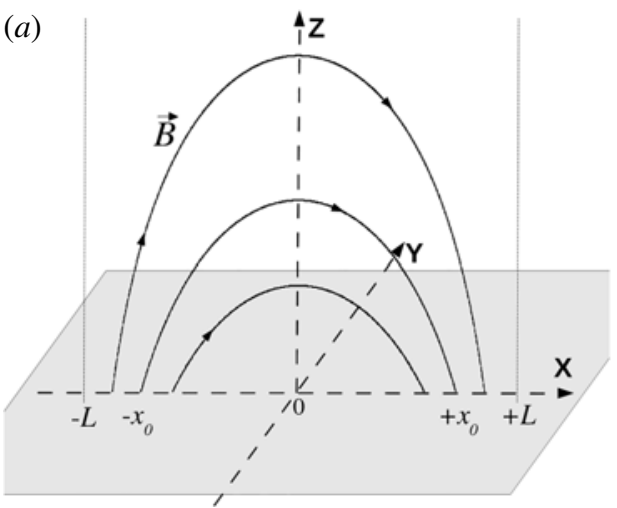

(b)

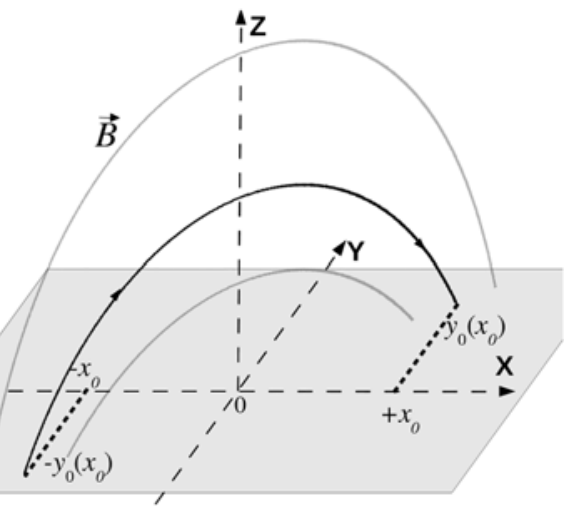

FIgURE 4. Coronal magnetic arcade. (a) Potential magnetic field $(\alpha=0) ;(b)$ shearing of magnetic field lines $(\alpha \neq 0)$.

form when $B_{y}(\Psi)=\alpha \Psi$ with $\alpha=$ const. (the so-called linear force-free magnetic field). In this case the flux function $\Psi$ satisfies the linear equation

$$
\nabla^{2} \Psi+\alpha^{2} \Psi=0
$$

Consider now a linear force-free field that does not vary along $y$-axis and corresponds to the flux function

$$
\Psi(x, z)=\frac{2 B_{0} L}{\pi} \cos \left(\frac{\pi x}{2 L}\right) \exp (-\kappa z), \quad \kappa=\frac{\pi}{2 L} \sqrt{1-\frac{4 \alpha^{2} L^{2}}{\pi^{2}}}>0 .
$$

(It is easy to verify that this $\Psi$ does satisfy (3.4).) In the 2 -D domain $\{-L \leqslant x \leqslant$ $L, z>0\}$ such a field can be used as a model of the bipolar coronal magnetic arcade located above the photospheric surface $z=0$ (see figure 4). When $\alpha=0$, the field is a potential one with the magnetic field lines lying in the $(x, z)$-plane $\left(B_{y} \equiv 0\right)$ (figure $4 a$ ). In the non-potential case, when $0<\alpha<\pi / 2 L$, the field component $B_{y}(x, z)=\alpha \Psi(x, z)$ is present, which makes the magnetic field lines stretch along the $y$-axis as shown in figure $4(b)$. Another effect of the non-potentiality of the arcade is its vertical bulging. An increase in $\alpha$ makes the parameter $\kappa$ in (3.5) smaller, so the arcade extends further upward. However, note that whatever a magnitude of $\alpha$, 
the magnetic field defined by (3.5) has the same unchanged normal component at the boundary of the arcade domain. Thus, $\left.B_{n}\right|_{z=0}=\left.B_{z}\right|_{z=0}=\partial \Psi /\left.\partial x\right|_{z=0}=-B_{0} \sin (\pi x / 2 L)$, while $B_{n}=0$ at $x= \pm L$ and at $z \rightarrow+\infty$. Therefore, as discussed in $\S 2$, the evolution of the magnetic energy in the arcade, $W_{M}$, can be considered independently of its exterior. This energy, calculated per unit length along the $y$-axis, is given by the integral $W_{M}=\int_{-L}^{+L} \mathrm{~d} x \int_{0}^{+\infty} \mathrm{d} z B^{2} / 8 \pi$. Then, according to (3.1) and (3.5), the magnetic field components are equal to

$$
\begin{gathered}
B_{x}=-\frac{\partial \Psi}{\partial z}=B_{0} \sqrt{1-\frac{4 \alpha^{2} L^{2}}{\pi^{2}}} \cos \left(\frac{\pi x}{2 L}\right) \mathrm{e}^{-\kappa z}, \\
B_{y}=\alpha \Psi=B_{0} \frac{2 \alpha L}{\pi} \cos \left(\frac{\pi x}{2 L}\right) \mathrm{e}^{-\kappa z}, \\
B_{z}=\frac{\partial \Psi}{\partial x}=-B_{0} \sin \left(\frac{\pi x}{2 L}\right) \mathrm{e}^{-\kappa z}
\end{gathered}
$$

and a straightforward integration yields

$$
W_{M}=\frac{B_{0}^{2} L^{2}}{4 \pi^{2}}\left(1-\frac{4 \alpha^{2} L^{2}}{\pi^{2}}\right)^{-1 / 2} .
$$

As expected, this magnetic energy is minimal for the potential magnetic field with $\alpha=0$, so the excess magnetic energy of the arcade is equal to

$$
\Delta W_{M}=W_{M}(\alpha)-W_{M}(\alpha=0)=\frac{B_{0}^{2} L^{2}}{4 \pi^{2}}\left[\left(1-\frac{4 \alpha^{2} L^{2}}{\pi^{2}}\right)^{-1 / 2}-1\right] .
$$

The excess magnetic energy is supplied to the corona by photospheric motions (see §1). Therefore, consider now in more detail the energy balance of this process for the model under discussion. In this case, a signature of the field non-potentiality is its non-zero toroidal component $B_{y}$ (and, hence, extension of field lines along the $y$-axis). Therefore, the first step is to derive the relation between the non-potentiality parameter $\alpha$ and the magnetic footpoints displacement in this direction, $\pm y_{0}\left(x_{0}\right)$, as shown in figure 4(b). To do so, we define each magnetic field line of the arcade by the parameter $x_{0}$, which is the $x$-coordinate of its footpoints on the photospheric surface $z=0$. In a non-potential field these footpoints are separated in $y$-direction by a distance $\Delta y$, which can be calculated by using expressions (3.6) for the field components as the following integral along a field line $\Psi(x, z)=$ const.:

$$
\Delta y=\int_{-x_{0}}^{+x_{0}} \mathrm{~d} y=\int_{-x_{0}}^{+x_{0}} \frac{B_{y}}{B_{x}} \mathrm{~d} x=x_{0} \frac{4 \alpha L}{\pi}\left(1-\frac{4 \alpha^{2} L^{2}}{\pi^{2}}\right)^{-1 / 2} .
$$

Since

$$
\Delta y=2 y_{0}\left(x_{0}\right), \quad \text { it implies that } y_{0}\left(x_{0}\right)=x_{0} \frac{2 \alpha L}{\pi}\left(1-\frac{4 \alpha^{2} L^{2}}{\pi^{2}}\right)^{-1 / 2} .
$$

Assume now that such a displacement is provided by shearing flow $V_{y}^{(p h)}(x)$ at the photospheric surface, which forces the magnetic arcade to evolve through a sequence 
of the force-free equilibria of the form (3.5), with the parameter $\alpha$ varying with time as some function $\alpha(t)$. This requires that

$$
V_{y}^{(p h)}\left(x_{0}\right)=\frac{\mathrm{d} y_{0}\left(x_{0}\right)}{\mathrm{d} t}=x_{0} \frac{2 L}{\pi} \frac{\mathrm{d}}{\mathrm{d} t}\left[\alpha\left(1-\frac{4 \alpha^{2} L^{2}}{\pi^{2}}\right)^{-1 / 2}\right]=x_{0} \frac{2 L}{\pi}\left(1-\frac{4 \alpha^{2} L^{2}}{\pi^{2}}\right)^{-3 / 2} \frac{\mathrm{d} \alpha}{\mathrm{d} t} .
$$

On the other hand, temporal variation of $\alpha$ means that the free magnetic energy (3.8) stored in the arcade is also changing with time. As seen from (3.8), this energy becomes increased for larger $\alpha$ in the course of the photospheric shearing deformation of the field. Since electric resistivity of the hot coronal plasma is very low (see $\S 4$ ), the time scale of the magnetic relaxation to a potential ground-energy state is very long. Therefore, during the phase of the magnetic energy storage in the corona, Ohmic dissipation of the magnetic energy is small, so coronal plasma can be considered a perfectly conducting medium with no Ohmic energy losses. Furthermore, under the quasistatic evolution of the arcade, plasma velocity remains highly sub-Alfvénic, so kinetic energy of the plasma flow is small compared to the magnetic energy of the system. Therefore, the energy conservation law requires that the rate of change of the excess magnetic energy (3.8) should be equal to the energy flux supplied to the arcade by the photospheric flow. Since the latter is determined by the Poynting flux $\boldsymbol{P}=c(\boldsymbol{E} \times$ B) $/ 4 \pi$, the necessary requirement reads

$$
\frac{\mathrm{d}\left(\Delta W_{M}\right)}{\mathrm{d} t}=\left.\int_{-L}^{+L} P_{z}\right|_{z=0} \mathrm{~d} x
$$

In a moving perfectly conducting fluid, the electric field is equal to $\boldsymbol{E}=-(\boldsymbol{V} \times \boldsymbol{B}) / c$ (see, e.g. Landau, Lifshitz \& Pitaevskii 1984), and the surface integral in (3.12) can be written as

$$
\left.\int_{-L}^{+L} P_{z}\right|_{z=0} \mathrm{~d} x=\frac{1}{4 \pi} \int_{-L}^{+L} \mathrm{~d} x\left[V_{z} B^{2}-B_{z}(\boldsymbol{V} \cdot \boldsymbol{B})\right]_{z=0}=-\frac{1}{4 \pi} \int_{-L}^{+L} \mathrm{~d} x V_{y}^{(p h)}\left(B_{y} B_{z}\right)_{z=0} .
$$

By using expressions (3.6) and (3.11) for the magnetic field and the photospheric velocity, a straightforward calculation of this integral yields

$$
\int_{L}^{+L} \mathrm{~d} x P_{z}(z=0)=\frac{B_{0}^{2} L^{4}}{\pi^{4}} \alpha\left(1-\frac{4 \alpha^{2} L^{2}}{\pi^{2}}\right)^{-3 / 2} \frac{\mathrm{d} \alpha}{\mathrm{d} t},
$$

which, according to (3.8), satisfies the energy conservation requirement (3.12). Thus, it completes the demonstration of how mechanical energy of the granular photospheric flow is transformed into the free magnetic energy stored in the solar corona.

\section{Energy release: a brief summary}

During a fairly strong solar flare, energy of the order of $(\Delta W)_{f} \sim 10^{32} \mathrm{erg}$ is released in a matter of tens of minutes (see, e.g. Schrijver 2009), i.e. $(\Delta t)_{f} \sim 10^{3} \mathrm{~s}$. As this energy is tapped from the free magnetic energy stored in the corona, one can estimate what coronal volume $(\Delta V)_{f}$ should be involved in such an event. Thus, assuming that a respective coronal structure is substantially non-potential, i.e. its non-potential field component $\boldsymbol{b}$ (see $\S 2$ ) is of the order of $B_{c} \sim 10^{2} \mathrm{G}$, one gets from (2.5) that $b^{2} / 8 \pi(\Delta V)_{f} \sim B_{c}^{2} / 8 \pi(\Delta V)_{f} \sim(\Delta W)_{f}$, which yields $(\Delta V)_{f} \sim 3 \times 10^{28} \mathrm{~cm}^{3}$, 
and, hence, the scale length $L_{f} \sim(\Delta V)_{f}^{1 / 3} \sim 3 \times 10^{9} \mathrm{~cm}$. It looks quite reasonable since such $L_{f}$ is comparable to a typical loop length $L_{c}$ in a coronal active region. There is, however, a striking disparity between the observed flare time $(\Delta t)_{f}$ and the time $\tau_{\eta}$ of the global resistive magnetic energy dissipation in the corona. If one assumes that the non-potential field component $\boldsymbol{b}$ varies on a length scale of the order of $L_{c}$, the electric current in the corona can be estimated as $\boldsymbol{j}_{\left(L_{c}\right)}=c(\nabla \times \boldsymbol{b}) / 4 \pi \sim c b / 4 \pi L_{c}$. The volumetric power of Ohmic dissipation is equal to $Q_{\left(L_{c}\right)}=\eta j_{\left(L_{c}\right)}^{2} \sim \eta c^{2} b^{2} / 16 \pi^{2} L_{c}^{2}$ and, hence, it yields the respective dissipation time of

$$
\tau_{\eta} \sim \frac{b^{2} / 8 \pi}{Q} \sim \frac{L_{c}^{2}}{D_{\eta}}, \quad \text { where } D_{\eta} \equiv \frac{\eta c^{2}}{4 \pi}
$$

is the so called resistive magnetic diffusivity of a medium. In a hot fully ionized plasma this magnetic diffusivity scales as $D_{\eta} \propto T^{-3 / 2}$, and at the coronal temperature of $T_{c} \sim 10^{6} \mathrm{~K}$ it becomes equal to $D_{\eta} \approx 10^{4} \mathrm{~cm}^{2} \mathrm{~s}^{-1}$. Thus, for $L_{c} \sim 10^{9} \mathrm{~cm}$ it yields the global dissipation time $\tau_{\eta} \approx 10^{14}$ s (i.e. $\approx 3$ million years!), which is clearly completely irrelevant.

It should be noted, however, that such a long time is required for a complete magnetic relaxation to the ground-energy state with a potential magnetic field. Instead, a fraction (typically, a substantial one) of the excess magnetic energy (2.5) can be released much faster via the process of magnetic reconnection (Yamada, Kulsrud \& Ji 2010). In this case, the electric current is not distributed smoothly throughout the whole relaxation domain (as it was assumed in the estimation of $j_{c}$ given above) but is strongly concentrated inside some small regions with a spatial scale $l \ll L_{c}$. In these regions, called 'current sheets', the electric current density $j_{l}$ is much higher than $j_{L_{c}}, j_{l} \sim j_{L_{c}}\left(L_{c} / l\right) \gg j_{L_{c}}$. Therefore, the role of even a weak resistivity becomes significantly enhanced inside such current sheets, which results in a much faster breaking up of magnetic field lines. This local effect allows global restructuring of the magnetic field that brings about a partially relaxed state. The latter, being an equilibrium configuration in a low- $\beta$ plasma, should be a force-free magnetic field described by (2.1). Quite remarkably, it turns out that these partially relaxed states correspond to constant- $\alpha$ force-free fields for which the function $\alpha(\boldsymbol{r})=$ const. The reason is associated with a characteristic of the magnetic configuration known as global magnetic helicity $H=\int_{V} \boldsymbol{A} \cdot \boldsymbol{B} \mathrm{d} V$, where $\boldsymbol{A}$ is a vector potential of the magnetic field. This quantity, which is a measure of the twistedness and knottedness of the magnetic field (see, e.g. Pfister \& Gekelman 1991 and references therein), is approximately conserved under magnetic reconnection in small current sheets (Taylor 1974, 1986). Therefore, constant- $\alpha$ fields represent a minimum of the total magnetic energy under the constraint of a constant magnetic helicity (Woltjer 1958). Note that complete magnetic relaxation to a potential magnetic configuration, helicity of which is equal to zero, implies destruction of the magnetic helicity.

In the context of magnetic relaxation in the solar corona, a possible role of magnetic helicity and constant- $\alpha$ force-free fields has been first discussed in Norman \& Heyvaerts (1983). Since small-scale current sheets are likely to form quite readily in the solar corona (Parker 1972), magnetic reconnection seems to represent a viable mechanism that underlies solar coronal activity. There is also direct observational evidence of reconfiguration of the magnetic field in the corona, presumably due to magnetic reconnection, during solar flares (Tsuneta 1996).

Consider now the time scale $\tau_{R}$ of the partial magnetic relaxation via the current sheets reconnection. Here it is useful to introduce a non-dimensional parameter called 
the Lundquist number, which is defined as $S \equiv \tau_{\eta} / \tau_{A}=L V / D_{\eta}$. This is typically very large for high-temperature plasma applications in laboratory and space. For example, in fusion-oriented devices, $S \sim 10^{6}$, while in the solar corona $S_{c} \sim 10^{12}-10^{14}$. Thus, under $S \gg 1$, the classical Sweet-Parker model (Parker 1957; Sweet 1958) of the current sheet reconnection yields $\tau_{R} \sim \tau_{A} S^{1 / 2}$. Although this time is much shorter than the global resistive time $\tau_{\eta}=\tau_{A} S$ that is required for the complete magnetic relaxation, it is still far too long to account for what is actually observed. Moreover, note that the time scale of solar flares, which is $(\Delta t)_{f} \sim 10^{3} \mathrm{~s}$, is only approximately hundred times longer than the coronal Alfvén transit time $\tau_{A} \sim 10 \mathrm{~s}$. Therefore, bearing in mind the extremely large value of the Lundquist number $S_{c}$, one has to conclude that any realistic theoretical model of solar flares should yield the relaxation time which is virtually independent on a magnitude of the Lundquist number. This issue, called the problem of fast magnetic reconnection, is presently a hot research topic (see, e.g. Loureiro \& Uzdensky (2016) for its most recent developments).

\section{Acknowledgements}

The author is grateful to M. Gordovskyy for his help in preparing the figures for this article, as well as to G. Bendo, I. Browne, and P. Browning for a number of useful comments.

\section{REFERENCES}

Amari, T., Aly, J. J., Luciani, J. F., Boulmezaoud, T. Z. \& Mikic, Z. 1997 Reconstructing the solar coronal magnetic field as a force-free magnetic field. Solar Phys. 174, 129-149.

Golub, L. \& Pasachoff, J. 2010 The Solar Corona, 2nd edn. Cambridge University Press.

KANNENBERG, L. 1989 Uniqueness of solutions to Helmholtz's equation with linear boundary conditions. Am. J. Phys 57, 60-63.

Landau, L. D., Lifshitz, E. M. \& Pitaevskit, L. P 1984 Electrodynamics of Continuous Media, chap. 8. Pergamon Press.

Loureiro, N. F. \& Uzdensky, D. A. 2016 Magnetic reconnection: from the Sweet-Parker model to stochastic plasmoid chains. Plasma Phys. Control. Fusion 58, 014021.

Meyer-Vernet, N. 2007 Basics of the Solar Wind. Cambridge University Press.

Norman, C. A. \& Heyvaerts, J. 1983 The final state of a solar flare. Astron. Astrophys. 124, L1-L3.

PARKER, E. N. 1957 Sweet's mechanism for merging magnetic fields in conducting fluids. J. Geophys. Res. 62, 509-520.

PARKER, E. N. 1972 Topological dissipation and the small-scale fields in turbulent gases. Astrophys. J. 174, 499-510.

Pfister, H. \& GeKelman, W. 1991 Demonstration of helicity conservation during magnetic reconnection using Christmas ribbons. Am. J. Phys. 59, 497-502.

SChriJver, C. J. 2009 Driving major solar flares and eruptions: a review. Adv. Space Res. 43, 739-755.

SwEET, P. A. 1958 The neutral point theory of solar flares. In Electromagnetic Phenomena in Cosmical Physics, Proc. IAU Symp. 6, pp. 123-127. Cambridge University Press.

TAYLOR, J. B. 1974 Relaxation of toroidal plasmas and generation of reverse magnetic fields. Phys. Rev. Lett. 33, 1139-1141.

TAYlOR, J. B. 1986 Relaxation and magnetic reconnection in plasmas. Rev. Mod. Phys. 58, 741-763.

TSUnETA, S. 1996 Structure and dynamics of magnetic reconnection in a solar flare. Astrophys. J. 456, 840-849.

Woltjer, L. 1958 A theorem on force-free magnetic fields. Proc. Natl Acad. Sci. 44, 489-491.

Yamada, M., Kulsrud, R. \& Ji, H. 2010 Magnetic reconnection. Rev. Mod. Phys. 82, 603-664. 